\title{
Beam instability induced by space charge oscillation during final beam bunching for heavy ion inertial fusion
}

\author{
Takashi Kikuchi* \\ Center for Nuclear Study, Graduate School of Science, University of Tokyo, Wako-Branch at RIKEN, 2-1 Hirosawa, \\ Wako, Saitama, 351-0198, Japan \\ Mitsuo Nakajima and Kazuhiko Horioka \\ Department of Energy Sciences, Interdisciplinary Graduate School of Science and Engineering, Tokyo Institute of Technology, \\ Nagatsuta 4259, Midori-ku, Yokohama, 226-8502, Japan \\ Takeshi Katayama \\ Center for Nuclear Study, Graduate School of Science, University of Tokyo, Wako-Branch at RIKEN, 2-1 Hirosawa, \\ Wako, Saitama, 351-0198, Japan \\ and Beam Physics and Engineering Laboratory, Institute of Physical and Chemical Research (RIKEN), 2-1 Hirosawa, \\ Wako, Saitama, 351-0198, Japan
}

(Received 25 September 2003; published 31 March 2004)

\begin{abstract}
Beam instability excited by space charge effect is investigated by particle simulations during final beam bunching in heavy ion inertial fusion. The beam current is continuously increased along the beam transport line with longitudinal bunch compression. A transverse particle-in-cell simulation is carried out with a simple model of beam current increase. The multiparticle simulation shows emittance growth accompanied by the restructuring of charge distribution in the interior of the beam. An analytical stability criterion for the high-current beam transport predicts the growth of density fluctuation by the space charge effect. Although results indicate that an abrupt emittance growth is induced by the space charge oscillation at critical tune depression in the final buncher, the increase of emittance can be estimated as high as $23 \%$ at the worst case during the bunching process.
\end{abstract}

DOI: 10.1103/PhysRevSTAB.7.034201

PACS numbers: 29.27.Bd, 52.58.Hm, 52.65.Rr

\section{INTRODUCTION}

For a production of inertial fusion energy, a driver system based on heavy ion beams is one attractive option. In a heavy ion inertial fusion (HIF), the generation and transport of intense heavy ion beam are especially important issues. The required beam parameters are of the order of $\mathrm{GeV}$ particle energy, $100 \mathrm{kA}$ total current, and $10 \mathrm{~ns}$ pulse width for an effective implosion of a fuel pellet. A particle accelerator can produce the heavy ion beam with high particle energy. However these parameters in the HIF driver system have not yet been determined, because dynamics of such a space charge dominated beam might include a lot of unknown behaviors. The space charge dominated beam can be considered to be so-called "non-neutral plasmas" [1,2], and there has been increasing interest in the behavior of the intense ion beam in recent years [3]. Consequently, the study of highcurrent and a space charge dominated beam is crucial issue in HIF [2,4].

For space charge dominated beams, the stability of the beam transport has been investigated by theoretical analyses and numerical simulations [5-13]. From these investigations, it is indicated that instability induced by the space charge effect could cause a mismatch

*Electronic address: tkikuchi@cns.s.u-tokyo.ac.jp oscillation, a halo formation, emittance growth, and particle loss. Most of the analytical considerations have concentrated on Kapchinskij-Vladimirskij (KV) distribution [14] in transverse phase space. In a matched KV distribution beam, the stability analysis implies that the instability exists in tune depressions less than 0.4 [7]. Although for the distribution of the $\delta$ function in fourdimensional phase space the KV beam is useful for the analytical treatment; the distribution has a highly inverted population. For this reason, the stability analysis with the KV distribution often tends to overestimate the instability effect in comparison with a realistic beam. Recently, Startsev and Davidson [15] introduced an analytical treatment with a continuous sheet beam in a waterbag (WB) distribution [3] which is uniform in the phase space and has a more realistic distribution than the KV beam. In the analysis, it is found that the sheet beam WB distribution is manifestly stable under the condition of the matched beam transport. On the other hand, the KV and WB beams cause the instability under the space charge dominated beam transport with mismatch oscillations [10-12]. While their studies discuss the axisymmetric or nonaxisymmetric beam behavior in the continuous or periodic lattice, the beam current is fixed as a constant.

In the final stage of a HIF accelerator system, the beam pulse must be longitudinally compressed from $\sim 100$ to $\sim 10 \mathrm{~ns}$ [16-19]. For this purpose, induction 
voltage modulators, which have a precise waveform controllability and repetition capability of a few $\mathrm{MHz}$, are a useful device for the high-current beam control [20]. A beam buncher composed of the induction modulator applies bunching voltage so as to make a considerable velocity tilt between the head and the tail of the beam bunch [16-19,21]. Although the detailed system designs are not yet completed, the final beam bunching is indispensable in all of the suggested HIF driver systems [22]. For the effective pellet implosion of HIF, we should transport and compress the bunch of heavy ion beam without emittance growth and particle loss as much as possible. As a final focus and beam irradiation are crucial issues for the effective implosion of the HIF fuel pellet [23], large emittance interferes with the tight focusing to the small target. In a final beam bunching, the beam current is continuously increased due to the bunch compression, and the stability condition of the beam transport is also changed with the increase of the space charge effect.

We investigate the beam dynamics under a strong space charge effect during the longitudinal bunch compression in the final beam bunching of the HIF accelerator system. In previous studies $[24,25]$, the dilution of particle distribution in phase space has been implied by particle simulations during the longitudinal bunch compression. In this paper, the particle simulations show the emittance growth accompanied by localized charge distribution. The comparison with the analytical estimation for the growth rate of axisymmetric flute perturbation confirms an important role of space charge oscillation on the emittance growth.

\section{TRANSVERSE BEAM DYNAMICS SIMULATION USING THE PARTICLE-IN-CELL METHOD WITH A LONGITUDINAL BUNCH COMPRESSION MODEL}

In the final beam bunching of the HIF accelerator system, the high-current and high-energy beam is transported using a magnetic quadrupole focusing channel. Based on a strong focus theory $[1,3,26]$, we can construct the quadrupole channel as a form composed of focusdrift-defocus-drift (FODO) lattice [1,3]. Generally, the beam transport by FODO lattice with magnetic quadrupole causes a nonaxisymmetric behavior in the beam cross section. For numerical simulation studies, a large number of the calculation grids (mesh) should be prepared for observation of the physics with a Debye length scale. In addition, we are interested in the beam dynamics with longitudinal bunch compression, so that the simulation for beam dynamics requires a self-consistent fully threedimensional numerical scheme. However, such full calculations are quite difficult from the viewpoint of the computational cost for the present, i.e., calculation time and required memory size in the computer system.

While the longitudinal bunch length is of the order of a meter, the scale of the transverse cross section is only approximately a centimeter in the final beam bunching stage [27]. Consequently, the small-scale phenomena by the space charge structure will be dominated by the transverse beam dynamics. For this reason, we deal with the particle dynamics in the transverse cross section of the beam by multiparticle simulation, and the effect of longitudinal compression is introduced as the beam current increase [28].

The scale of the cross section and its space charge field should change with the longitudinal position in the beam bunch [19]. Although the lattice condition could be adjusted to a part of the beam cross section, a perfect matching condition throughout the whole cross section of the beam bunch is considered to be difficult to realize. In this study, we fix the lattice condition and concentrate our attention on the effect of the initial transverse distribution on the emittance evolution during the bunching process.

\section{A. Particle simulation implementations}

We use a particle-in-cell (PIC) method [29,30] for descriptions of the transverse behavior of the beam bunch with the longitudinal compression, the effect of which was evaluated by assuming a linearly increasing beam current model. In the PIC method, the single ion behavior is replaced as a superparticle one, so that the superparticle is a representative of many real ions. For the particle positions $x_{p}$ and $y_{p}$ of index $p$, the equations of motion along the transport distance $s$ are written as [31,32]

$$
\begin{gathered}
\frac{d^{2} x_{p}}{d s^{2}}=-k_{t} h x_{p}-\frac{q e}{\gamma^{3} m_{0} v_{z}^{2}} \frac{\partial \phi}{\partial x}, \\
\frac{d^{2} y_{p}}{d s^{2}}=k_{t} h y_{p}-\frac{q e}{\gamma^{3} m_{0} v_{z}^{2}} \frac{\partial \phi}{\partial y},
\end{gathered}
$$

where $k_{t}$ is the transverse confinement force by the FODO lattice, $h \equiv h(s)$ is an alternating periodic step function in the FODO lattice of period length $S$ as shown in Fig. 1,

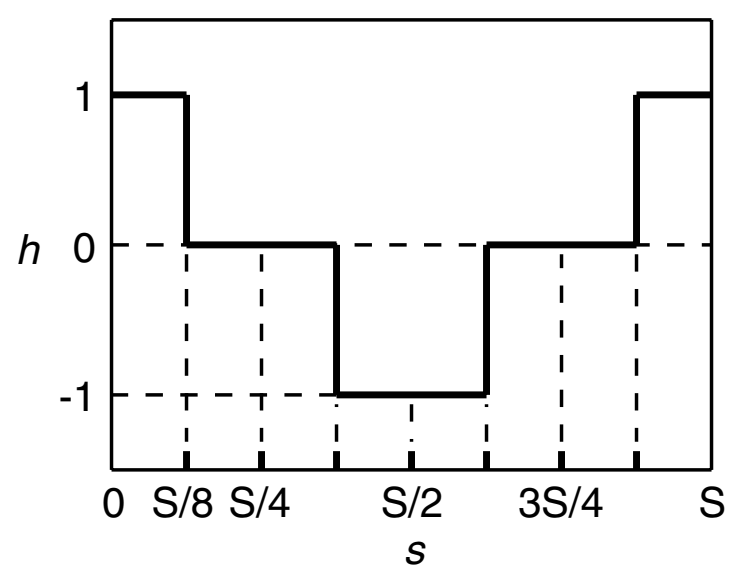

FIG. 1. Alternating periodic step function $h(s)=h(s+S)$ of beam transport channel by FODO lattice. 
$q$ is the charge state of the beam ion, $e$ is elementary charge, $m_{0}$ is the rest mass of the beam ion, $\gamma$ is the relativistic factor at the center of the longitudinal beam position, $v_{z}$ is the longitudinal beam velocity, and $\phi$ is the self-electrostatic potential of the beam, respectively.

In this study, we simply assume the effect of the selfmagnetic field as a factor of $1 / \gamma^{2}[26,32,33]$. The selfelectrostatic potential is calculated by

$$
\frac{\partial^{2} \phi}{\partial x^{2}}+\frac{\partial^{2} \phi}{\partial y^{2}}=-\frac{\rho}{\epsilon_{0}},
$$

where $\rho$ is the (area) charge density given by the beam particle positions and $\epsilon_{0}$ is the permittivity of free space, respectively. The above Poisson equation (3) can be solved with the multigrid method using the successive overrelaxation solver [34,35].

The ratio per unit length of the real ion number to the superparticle number $N_{b} / N_{\mathrm{sp}}$ is initially given by

$$
\left(\frac{N_{b}}{N_{\mathrm{sp}}}\right)_{i}=\frac{I_{b}}{q e v_{z} N_{\mathrm{sp}}},
$$

where $N_{b}$ is the ion number in a beam bunch, $N_{\mathrm{sp}}$ is the particle number for simulation (i.e., number of superparticle), and $I_{b}$ is the beam current, respectively. The subscript $i$ means initial condition. Considering the longitudinal bunch compression, the ratio $N_{b} / N_{\mathrm{sp}}$ increases linearly during the transport as

$$
\frac{N_{b}}{N_{\mathrm{sp}}}=\left(\frac{R_{\mathrm{cf}}-1}{L_{t}} s+1\right)\left(\frac{N_{b}}{N_{\mathrm{sp}}}\right)_{i}
$$

where $R_{\mathrm{cf}}$ is the final compression ratio, and $L_{t}$ is the total length of the beam buncher which consists of the FODO lattice with induction modulators, respectively. According to Eq. (5), the charge and mass of the superparticles are increased during the final beam bunching, and the ratio $N_{b} / N_{\mathrm{sp}}$ performs the reweighting of the superparticle with the beam transport [28].

The parameters of one beam bunch were assumed as in Table I [36].

We must adapt the transverse beam confinement system to the above beam parameters for the stable beam transport. The FODO lattice parameters are related by [3]

TABLE I. Parameters of one beam for the final bunching stage in HIF [36].

\begin{tabular}{lc}
\hline \hline Ion species & $\mathrm{Pb}^{+1}$ \\
Particle energy & $10 \mathrm{GeV}$ \\
Beam current & $400 \mathrm{~A} \rightarrow 10 \mathrm{kA}$ \\
Pulse duration & $250 \mathrm{~ns} \rightarrow 10 \mathrm{~ns}$ \\
Ion number & $6.25 \times 10^{14}$ \\
\hline \hline
\end{tabular}

$$
\begin{aligned}
\cos \sigma_{0}= & \cos \theta \cosh \theta+\frac{L}{\ell} \theta(\cos \theta \sinh \theta-\sin \theta \cosh \theta) \\
& -\frac{1}{2}\left(\frac{L}{\ell}\right)^{2} \theta^{2} \sin \theta \sinh \theta
\end{aligned}
$$

and

$$
k_{t}=\left(\frac{\theta}{\ell}\right)^{2}
$$

where $\sigma_{0}$ is the phase advance per one lattice period without space charge, $\ell$ is the length of the quadrupole lens space ( $\mathrm{F}$ and/or $\mathrm{D}$ in FODO), $L$ is the length of the drift space (O in FODO), and $\theta$ is the focusing strength of the magnetic quadrupole lens, respectively. The phase advance is selected as $\sigma_{0}=72^{\circ}$ for avoiding unstable transport in space charge dominated beams [37]. As the policy of the smaller beam radius, we choose $k_{t}=$ $1.282082 \mathrm{~m}^{-2}$ under the conditions of $\ell=L=0.75 \mathrm{~m}$ by Eqs. (6) and (7). In this paper, the transport distance is assumed as $L_{t}=450 \mathrm{~m}[24,27]$, and the final compression ratio is $R_{\mathrm{cf}}=25$ as shown in Table I [36].

The number of total simulation particles $N_{\mathrm{sp}}$ is from $5 \times 10^{4}$ to $1 \times 10^{6}$. The superparticles are initially placed with KV or WB distributions [38,39]. For the initial particle arrangement, we use the Mersenne Twister pseudorandom number generator [40].

The transverse calculation region is fixed at $10 \mathrm{~cm} \times$ $10 \mathrm{~cm}$, and the boundary condition is given as a conductor wall, i.e., $\phi=0$ at the edges of the calculation region. To confirm the spatial resolution, we have tested from $64 \times$ 64 to $1024 \times 1024$ mesh numbers for the solver of the Poisson equation in two-dimensional transverse space. Debye length is an important physical parameter for the evaluation of collective effects in the space charge dominated beam. In two-dimensional WB distribution, the Debye length $\lambda_{\mathrm{D}}$ is approximately expressed by [41]

$$
\frac{\lambda_{\mathrm{D}}}{r_{b}} \sim \frac{1}{2} \frac{\nu}{\nu_{0}},
$$

where $r_{b}$ is the beam edge radius, and $\nu / \nu_{0}$ is tune depression which is the ratio of the wave number of transverse particle motion (betatron oscillation) with and without space charge, respectively. For the resolution of the Debye length scale, it is recommended that the calculation mesh size is set as $0.23 \lambda_{\mathrm{D}}[29,30]$. As a result, the mesh number over $256 \times 256$ is required at the final state of the parameters in Table I. Test calculations are carried out, and the results also ensure the criterion of mesh size based on the above expression in the Debye length. For this reason, we use $512 \times 512$ meshes for the accurate simulation.

\section{B. Results of particle simulation}

At first, we show the calculation results as the contour maps of charge distribution. Figure 2 shows the charge 


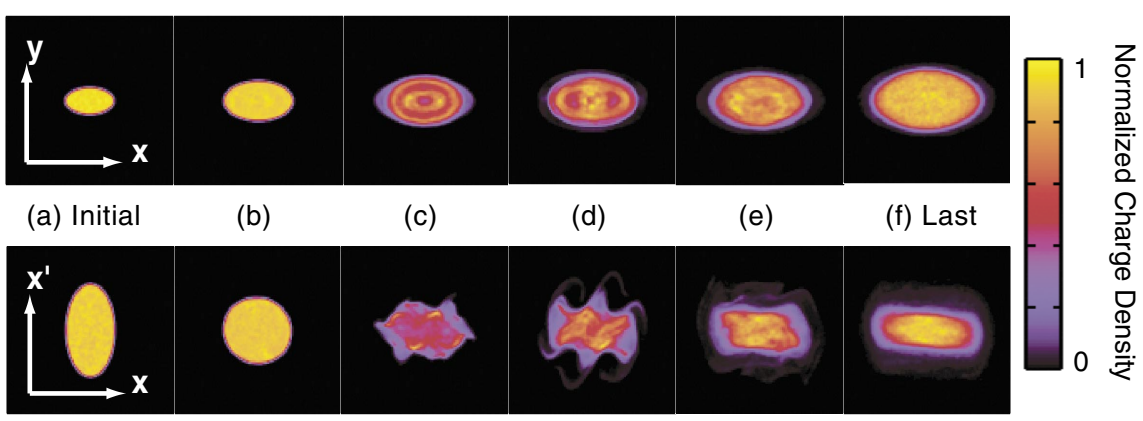

FIG. 2. (Color) Normalized charge density maps in real (top panels) and phase (bottom panels) spaces during transport with increasing beam current for initially KV distributed beam, (a) at initial condition, (b) at 50 lattice periods, (c) at 87 lattice periods, (d) at 96 lattice periods, (e) at 108 lattice periods, and (f) at 150 (last) lattice periods, respectively. The width and height correspond to $10 \mathrm{~cm} \times 10 \mathrm{~cm}$ in real space maps, and $10 \mathrm{~cm} \times 10 \mathrm{mrad}$ in phase space maps. The charge density in the contour map is normalized by the maximum value at each lattice period, and the value is expressed by the bar of the color level placed at the right side.

density maps in physical and phase spaces at each lattice period for the initially KV distributed beam.

In the real space maps, the horizontal and vertical scales correspond to the full calculation region, i.e., $10 \mathrm{~cm} \times 10 \mathrm{~cm}$. The charge density is normalized by the maximum value at each map (refer to a bar of the color level placed at the right side in the figure). The beam radius is extended with the beam current increase due to the longitudinal bunch compression. As shown in Fig. 2, localized charge distribution in real space and the dilution of the beam particle in phase space are indicated during the final beam bunching. Figure 3 shows the charge density maps in real and phase spaces for the initially WB distributed beam.

In WB distribution, qualitatively similar behaviors with the KV beam are observed as shown in Fig. 3. In these cases, the lattice parameters are fixed during the beam transport, so that the mismatch oscillations are not suppressed to zero.
Next, the emittance growth during the bunch compression is discussed from the particle simulation results. The root-mean-square (rms) transverse emittances are defined by

$$
\begin{aligned}
& \boldsymbol{\epsilon}_{x, \mathrm{rms}}=\left[\left\langle x^{2}\right\rangle\left\langle x^{\prime 2}\right\rangle-\left\langle x x^{\prime}\right\rangle^{2}\right]^{1 / 2}, \\
& \boldsymbol{\epsilon}_{y, \mathrm{rms}}=\left[\left\langle y^{2}\right\rangle\left\langle y^{\prime 2}\right\rangle-\left\langle y y^{\prime}\right\rangle^{2}\right]^{1 / 2},
\end{aligned}
$$

where each second moment is given by [42]

$$
\begin{aligned}
& \left\langle x^{2}\right\rangle=\frac{1}{N_{\mathrm{sp}}} \sum_{p=1}^{N_{\mathrm{sp}}} x_{p}^{2}, \\
& \left\langle y^{2}\right\rangle=\frac{1}{N_{\mathrm{sp}}} \sum_{p=1}^{N_{\mathrm{sp}}} y_{p}^{2},
\end{aligned}
$$

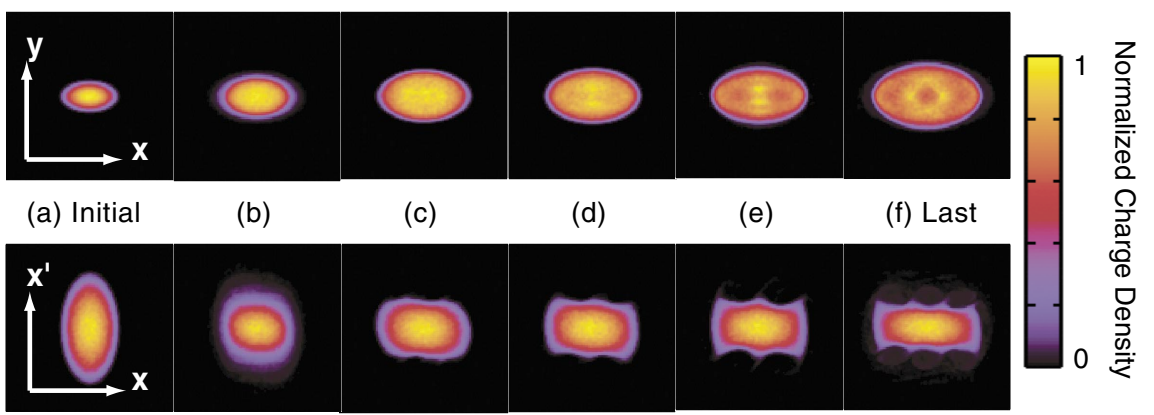

FIG. 3. (Color) Normalized charge density maps for real (top panels) and phase (bottom panels) spaces during transport with increasing beam current for initially WB distributed beam, (a) at initial condition, (b) at 50 lattice periods, (c) at 87 lattice periods, (d) at 96 lattice periods, (e) at 108 lattice periods, and (f) at 150 (last) lattice periods, respectively. The width and height correspond to $10 \mathrm{~cm} \times 10 \mathrm{~cm}$ in real space maps, and $10 \mathrm{~cm} \times 10 \mathrm{mrad}$ in phase space maps. The charge density in the contour map is normalized by the maximum value at each lattice period, and the value is expressed by the bar of the color level placed at the right side. 


$$
\begin{aligned}
\left\langle x^{\prime 2}\right\rangle & =\frac{1}{N_{\mathrm{sp}}} \sum_{p=1}^{N_{\mathrm{sp}}} x_{p}^{\prime 2}, \\
\left\langle y^{\prime 2}\right\rangle & =\frac{1}{N_{\mathrm{sp}}} \sum_{p=1}^{N_{\mathrm{sp}}} y_{p}^{\prime 2}, \\
\left\langle x x^{\prime}\right\rangle & =\frac{1}{N_{\mathrm{sp}}} \sum_{p=1}^{N_{\mathrm{sp}}} x_{p} x_{p}^{\prime}, \\
\left\langle y y^{\prime}\right\rangle & =\frac{1}{N_{\mathrm{sp}}} \sum_{p=1}^{N_{\mathrm{sp}}} y_{p} y_{p}^{\prime} .
\end{aligned}
$$

Here the prime denotes $d / d s$. We define the average of transverse rms emittances as $\epsilon=\left(\epsilon_{x, \mathrm{rms}}+\epsilon_{y, \mathrm{rms}}\right) / 2$ and assume the initial $\mathrm{rms}$ emittance to be $\epsilon_{i}=\epsilon_{x, \mathrm{rms}}=$ $\epsilon_{y, \mathrm{rms}}=10 \mathrm{~mm}$ mrad. Figure 4 shows the evolution of the emittance growth ratio $\epsilon / \epsilon_{i}$, which indicates the ratio of the average emittance to the initial one at each lattice period.

The simulation results of the beam transport with and without the increase of the beam current are shown in Fig. 4, which implies that the emittance is maintained during the beam transport without the compression. In the case of the KV beam, the radius is gradually diffused with the increasing beam current, but the emittance has not changed until 80 lattice periods in Fig. 4. As a result, the beam bunch is transported under the adiabatic matching condition down to the 80 lattice periods, even in the periodic lattice with constant lattice parameters and the continuous increase of the beam current. However, as shown in Fig. 4, the emittance abruptly increases after 80 lattice periods. Figure 5 shows the history of the

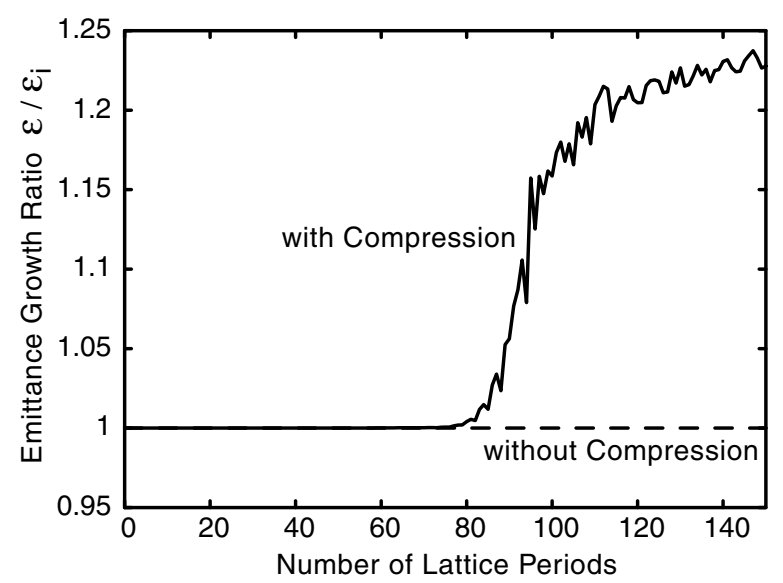

FIG. 4. Evolution of the emittance growth ratio with the initially KV distributed beam. Solid curve shows the emittance growth ratio with the beam current increase due to the longitudinal compression, and the dashed line indicates one without the compression.

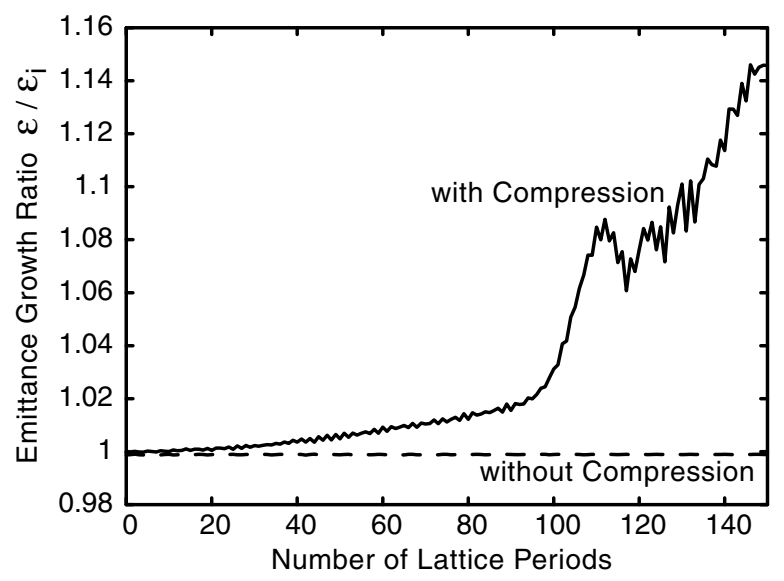

FIG. 5. History of emittance growth with initially WB distributed beam. Solid curve shows the emittance growth ratio with the beam current increase due to the longitudinal compression, and the dashed line indicates one without the compression.

emittance growth with and without longitudinal bunch compression in initial WB distribution.

In contrast to the KV distributed beam, continuous emittance growth occurs until 90 lattice periods, probably due to the small mismatch induced by the current increase. Similar to the result of the KV distribution, the emittance steeply increased over 90 lattice periods as shown in Fig. 5. After the longitudinal compression of the beam bunch, final emittance growth ratios at 150 lattice periods are around 1.23 and 1.15 in the initially $\mathrm{KV}$ and WB distributed beams, respectively.

\section{ANALYTICAL EVALUATION OF INSTABILITY INDUCED BY SPACE CHARGE OSCILLATION}

Unstable beam transport is caused by the instability due to the strong space charge effect $[1,7,9,10]$. As mentioned earlier, the beam physics is dominated by the space charge effect rather than the thermal motion in the beam transport line for the HIF driver system. The space charge dominated beam behaves as non-neutral plasma and can shield the external potential for the transverse beam confinement $[1,5,41]$. The collective behavior causes the restructuring of the space charge distribution. In the previous section, we showed that localized particle distribution in the beam interior is observed in the charge distribution maps, and the abrupt increase of emittance is also confirmed. In this section, we investigate theoretically the instability induced by the strong space charge effect, and previous simulation results are compared to the analytical consideration.

An unstable condition caused by the growth of the azimuthally symmetric flute perturbation is one possible instability due to the space charge effect [9-13]. The perturbed density of the beam interior 
produces the fluctuation of the self-electrostatic potential. If the amplitude of the potential perturbation grows above a threshold level during the beam transport, the localized space charge field generates significant nonlinear force, thereby inducing a mismatched oscillation and increase of emittance. Also, the mismatch oscillations of the beam radius cause the further emittance growth. The Vlasov-Poisson equations with KV distribution can predict the growth rate of the axisymmetric flute perturbation [1,9-13].

The instability condition has the threshold value on strength of the space charge effect. The tune depression is often used as the figure of merit of the transportability of space charge dominated beams. The tune depression $\nu / \nu_{0}$ (same as $\sigma / \sigma_{0}$ in the periodic lattice case) is given by $[10,11]$

$$
\frac{\nu}{\nu_{0}}=\sqrt{1+\frac{\alpha^{2}}{4}}-\frac{\alpha}{2}
$$

Here

$$
\alpha=\frac{K}{4 \epsilon_{n} \sqrt{k_{t}}},
$$

$K$ is the perveance and $\epsilon_{n}=\beta \gamma \epsilon$ is the normalized rms emittance with $\beta=v_{z} / c$ defining the ratio of the longitudinal beam velocity and speed of light $c$, respectively. The perveance is defined by

$$
K=\frac{2}{\beta^{3} \gamma^{3}} \frac{I_{b}}{I_{0}}
$$

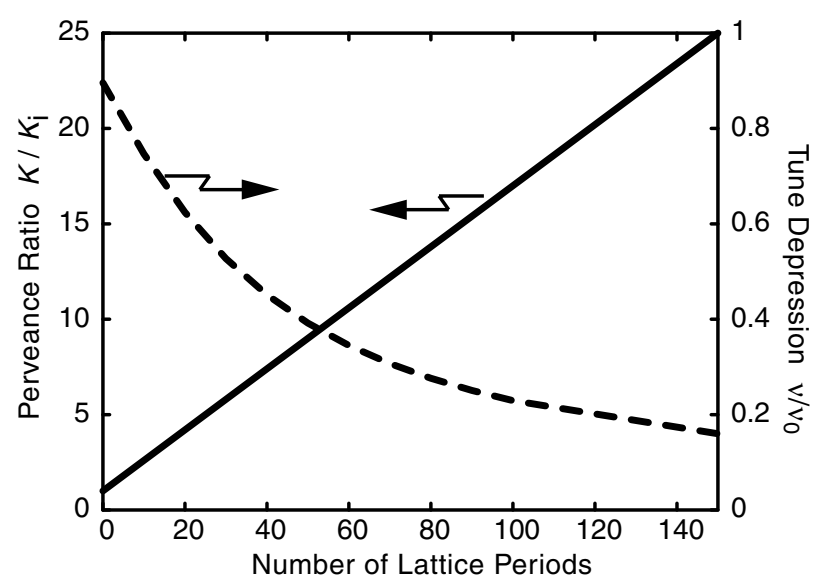

FIG. 6. Perveance ratio and tune depression as a function of transport lattice period. $K_{i}$ is initial perveance.

where $I_{0}=4 \pi \epsilon_{0} m_{0} c^{3} / q e$ is the characteristic current [3]. Equation (19) shows that the perveance is proportional to the beam current. Figure 6 shows the estimated tune depression from Eq. (17) during the perveance increase due to the longitudinal bunch compression.

The unnormalized rms emittance of $\epsilon=10 \mathrm{~mm} \mathrm{mrad}$ is assumed for the estimation. It is predicted that the tune depression will decrease from the initial 0.9 to 0.16 after the pulse compression.

At each mode number $m$, the growth rate of the axisymmetric flute perturbation can be evaluated by the dispersion relation in the case of the $\mathrm{KV}$ distribution $[10,11]$. The normalized mode frequency $\omega / \nu_{0}$ of the dispersion relation is calculated by [13]

$$
\left(\omega / \nu_{0}\right)^{4}-2\left[1+9\left(\nu / \nu_{0}\right)^{2}\right]\left(\omega / \nu_{0}\right)^{2}-4\left[1-17\left(\nu / \nu_{0}\right)^{2}\right]\left(\nu / \nu_{0}\right)^{2}=0
$$

in case of $m=2$. For $m=3$, the dispersion relation is written as

and

$$
\left(\frac{\omega}{\nu_{0}}\right)^{6}-2\left[1+27\left(\frac{\nu}{\nu_{0}}\right)^{2}\right]\left(\frac{\omega}{\nu_{0}}\right)^{4}+8\left[1+97\left(\frac{\nu}{\nu_{0}}\right)^{2}\right]\left(\frac{\nu}{\nu_{0}}\right)^{2}\left(\frac{\omega}{\nu_{0}}\right)^{2}-96\left[1+23\left(\frac{\nu}{\nu_{0}}\right)^{2}\right]\left(\frac{\nu}{\nu_{0}}\right)^{4}=0,
$$

$$
\begin{array}{r}
\left(\frac{\omega}{\nu_{0}}\right)^{8}-2\left[1+59\left(\frac{\nu}{\nu_{0}}\right)^{2}\right]\left(\frac{\omega}{\nu_{0}}\right)^{6}+52\left[1+83\left(\frac{\nu}{\nu_{0}}\right)^{2}\right]\left(\frac{\nu}{\nu_{0}}\right)^{2}\left(\frac{\omega}{\nu_{0}}\right)^{4}-32\left[19+1621\left(\frac{\nu}{\nu_{0}}\right)^{2}\right]\left(\frac{\nu}{\nu_{0}}\right)^{4}\left(\frac{\omega}{\nu_{0}}\right)^{2}- \\
288\left[9-521\left(\frac{\nu}{\nu_{0}}\right)^{2}\right]\left(\frac{\nu}{\nu_{0}}\right)^{6}=0
\end{array}
$$

in the case of $m=4$. Equations (20)-(22) indicate that the dispersion relation depends on the tune depression. The $m=4$ mode is the most serious, because it gives the largest threshold value of the tune depression $\nu / \nu_{0}$ [7]. In general, high-order instabilities are considered to be a consequence for the singularity of the KV distribution, so that the higher-order modes will not cause serious instabilities in realistic beams [1,13]. Although this is a formula for the continuous electrostatic focusing, it is often used to model the average focusing properties of an alternating-gradient lattice of magnetic quadrupoles $[1,3,13]$.
According to the tune depression given in Fig. 6, we can calculate the dispersion relation given by Eqs. (20)(22). The higher-degree equations of the dispersion relations can be solved using Bairstow's method [43]. Figure 7 shows the imaginary part $\operatorname{Im} \omega / \nu_{0}$ of the normalized mode frequency, which indicates the growth rate of each flute perturbation mode, during the final beam bunching.

As shown in Fig. 7, the growth rates in the cases of modes 2, 3, and 4 increase along the beam transport, because the tune depression is also decreased by the space 


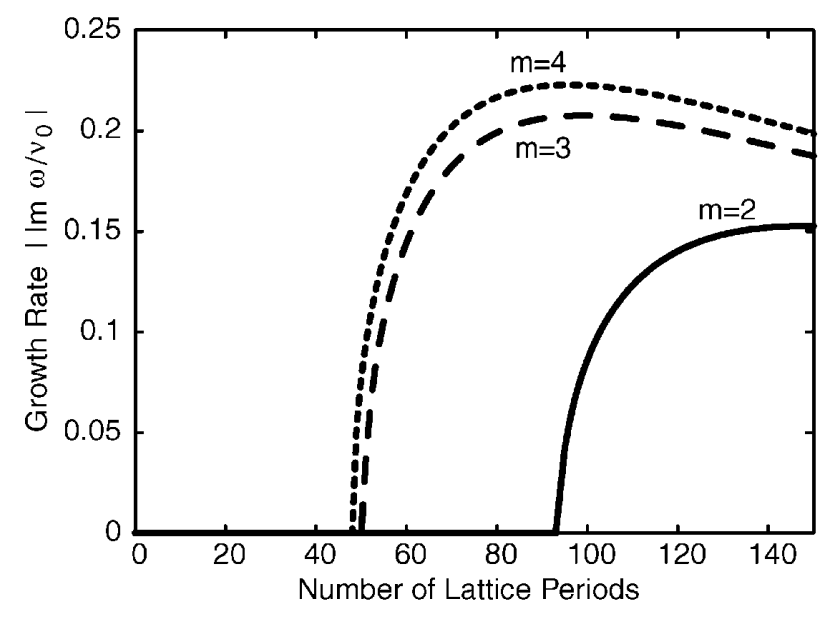

FIG. 7. Growth rate of axisymmetric flute perturbation as a function of transport lattice period for mode numbers $m=2,3$, and 4 during final beam bunching.

charge effect with the perveance increase. It is found that the emittance growth indicated by the previous section corresponds to the regime of the large growth rates of the axisymmetric flute perturbation.

Furthermore, we compare the perturbation of the charge density with the calculated one. The normalized body-wave density perturbation $\delta n / \delta n(r=0)$ for mode number $m=4\left(1-15 r^{2} / r_{b}^{2}+45 r^{4} / r_{b}^{4}-35 r^{6} / r_{b}^{6}\right.$, where $r$ and $r_{b}$ are the radius and the beam edge radius) [13] with highest growth rate is shown in Fig. 8.
Here the total density is expressed by $n=n_{0}+\delta n$ as the equilibrium density of $n_{0}$. On the other hand, we show a typical density perturbation given by the particle simulation with the initial $\mathrm{KV}$ beam as in Fig. 9.

The envelope radii $X$ and $Y$ can be calculated from $X=$ $2 \sqrt{\left\langle x^{2}\right\rangle}$ and $Y=2 \sqrt{\left\langle y^{2}\right\rangle}$, which are total beam radii in the $\mathrm{KV}$ distribution [3]. The envelope radii $X \approx Y \approx$ $1.9136 \mathrm{~cm}$ are given at $86+1 / 4$ lattice period. (According to the lattice configuration as shown in Fig. 1, the beam envelope $X$ is maximized at $s=S$ in one lattice period, while the envelope $Y$ has a minimum radius at $s=S$ as shown in Figs. 2 and 3. To this end, the envelope $X$ is equal to $Y$ at $s=S / 4$.) The perturbed density distribution is normalized by the difference from the average density in the beam. It is found that normalized perturbations obtained by the theoretical estimation shown in Fig. 8 and that by numerical simulation shown in Fig. 9 are similar. Consequently, it is suggested that the growth of the perturbed distribution inside the beam during the final bunching is induced by the instability. Although the analytical estimation assumes the $\mathrm{KV}$ distribution, the WB distribution also causes the instability in the mismatched beam transport [12]. These results suggest that the emittance growth observed in the multiparticle simulation is attributed to the instability due to the collective behavior in the space charge dominated beam.
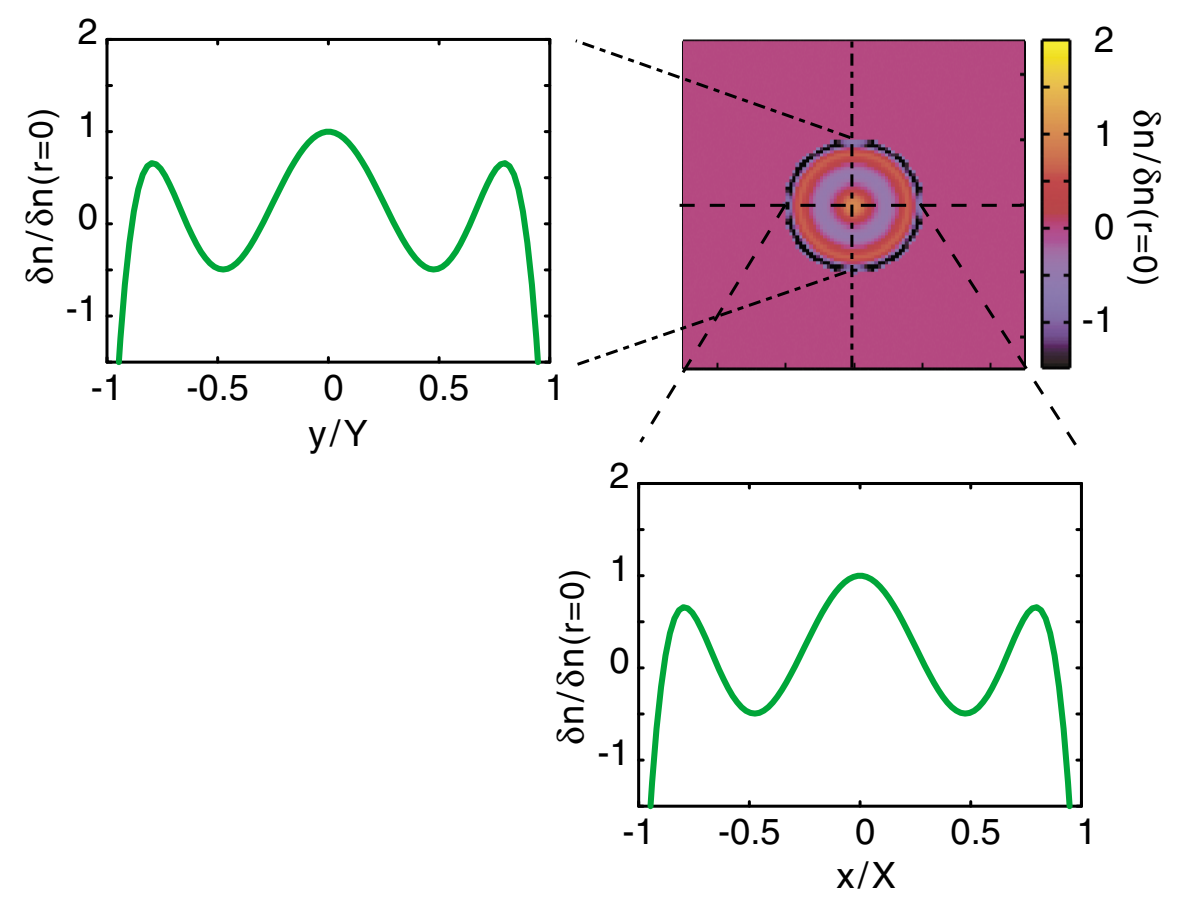

FIG. 8. (Color) Normalized density for flute perturbation in $m=4$ given by $1-15 r^{2} / r_{b}^{2}+45 r^{4} / r_{b}^{4}-35 r^{6} / r_{b}^{6}$. Contour map shows normalized perturbed density in transverse $(10 \mathrm{~cm} \times 10 \mathrm{~cm})$ real space. 

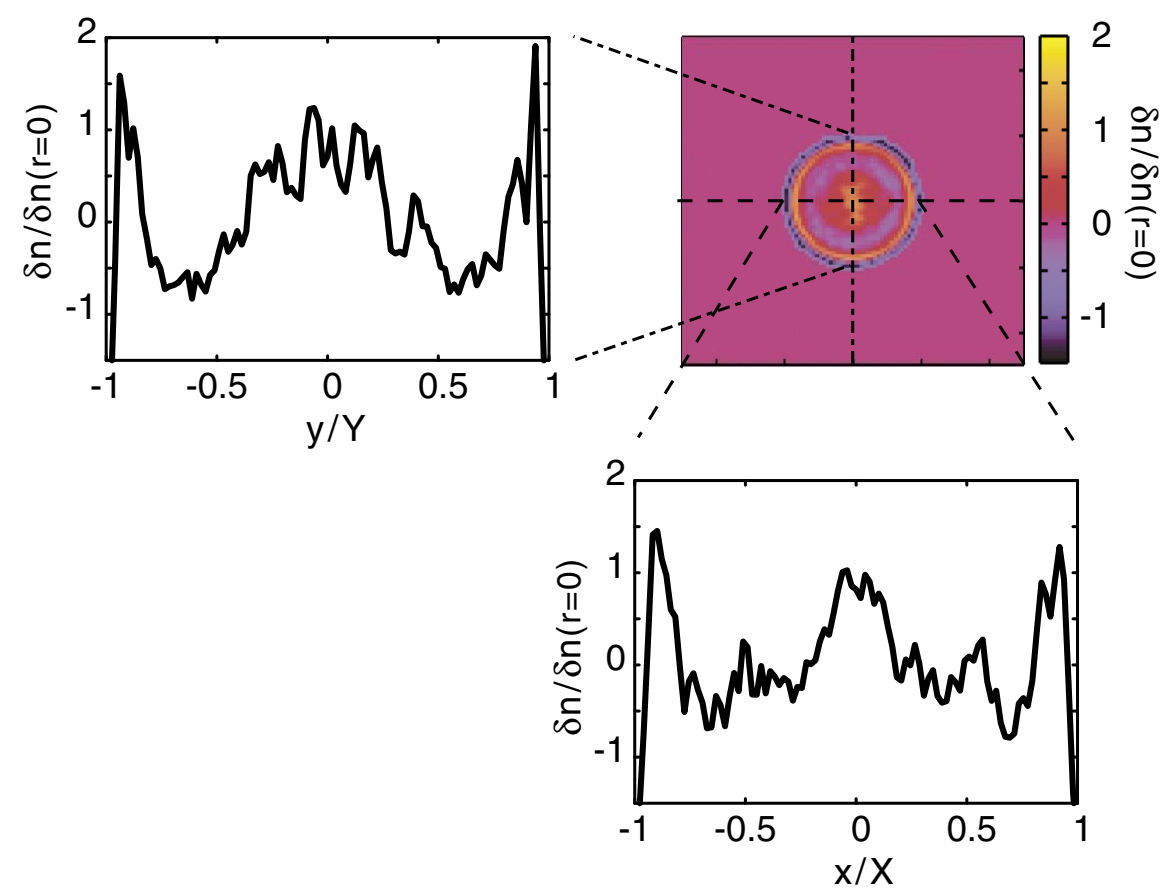

FIG. 9. (Color) Normalized perturbed density given by particle simulation in the case of the initial $\mathrm{KV}$ beam at $86+1 / 4$ lattice period. Contour map shows normalized perturbed density in transverse $(10 \mathrm{~cm} \times 10 \mathrm{~cm})$ real space. Envelope radii are $X=Y=$ $1.9136 \mathrm{~cm}$.

\section{CONCLUSION}

We investigated the transverse beam dynamics during the final beam bunching in the HIF accelerator system. Beam transverse PIC simulation with the increasing beam current, as a model of the longitudinal bunch compression, was carried out for the study of beam transport suffered from space charge oscillations.

The numerical simulation results indicated that the beam is bunched accompanied by the restructuring of charge density distribution and emittance growth during the final beam bunching and transport. For theoretical consideration, the growth rate of the axisymmetric flute perturbation was evaluated, and the comparison of the growth rate implied the unstable beam transport with the perturbation of $m=4$ mode during the final beam bunching. The regime of the large growth rate corresponded to the situation of the eventual emittance growth observed in the particle simulation. Although nonaxisymmetric instability modes were also implied by PIC results, the main cause of the emittance growth can be attributed to the instability excited by the strong space charge effect. Simulation results show that not only the KV distribution but also the WB beam undergoes the rapid emittance growth at around 80 lattice periods during the final transport. Analytical result also predicts that the space charge field significantly affects the beam transport in the parameter region of the HIF buncher under the condition with continuous increase of the beam current.

We summarize that the mechanism of the emittance growth during the final beam bunching is as follows: the space charge effect and mismatched oscillations cause the instability due to the space charge oscillation, and the density perturbations grow due to the instability. The nonuniform density distribution creates the nonlinear field, so that the emittance growth can be induced at the lattice position corresponding to the highest growth rate of the flute perturbation.

In this paper, particle simulations of the final beam bunching were carried out without adjusting lattice parameters along the beam current increase; nevertheless the emittance growth was $20 \%$ at the highest. We can conclude that even though the estimated emittance growth might not be negligible for the final focus and effective pellet irradiation, the space charge instability in the final buncher does not seriously affect the basic HIF concepts.

\section{ACKNOWLEDGMENTS}

A part of these calculation results was derived by the use of the Special Application Server B in RIKEN Computer and Information Division.

[1] R. C. Davidson, Physics of Nonneutral Plasmas (World Scientific, Singapore, 2001).

[2] J. J. Barnard, J. Fusion Energy 17, 223 (1998).

[3] M. Reiser, Theory and Design of Charged Particle Beams (Wiley, New York, 1994), and references therein. 
[4] M. Reiser, Fusion Eng. Des. 32-33, 133 (1996).

[5] I. Hofmann, Nucl. Instrum. Methods 187, 281 (1981).

[6] I. Hofmann, IEEE Trans. Nucl. Sci. 28, 2399 (1981).

[7] I. Hofmann, L. J. Laslett, L. Smith, and I. Haber, Part. Accel. 13, 145 (1983).

[8] I. Hofmann, G. Franchetti, O. Boine-Frankenheim, J. Qiang, and R. D. Ryne, Phys. Rev. ST Accel. Beams 6, 024202 (2003).

[9] R. L. Gluckstern, in Proceedings of the 1970 Proton Linear Accelerator Conference, Batavia, 1970 (National Accelerator Laboratory, Batavia, IL, 1971), p. 811.

[10] R. L. Gluckstern, W.-H. Cheng, and H. Ye, Phys. Rev. Lett. 75, 2835 (1995).

[11] R. L. Gluckstern, W.-H. Cheng, S. S. Kurennoy, and H. Ye, Phys. Rev. E 54, 6788 (1996).

[12] R. L. Gluckstern and S. S. Kurennoy, in Proceedings of the 1997 Particle Accelerator Conference, Vancouver, Canada (IEEE, Piscataway, NJ, 1998), p. 1950.

[13] S. M. Lund and R. C. Davidson, Phys. Plasmas 5, 3028 (1998).

[14] I. M. Kapchinskij and V.V. Vladimirskij, in Proceedings of the International Conference on High Energy Accelerators and Instrumentation (CERN, Geneva, 1959), p. 274.

[15] E. A. Startsev and R. C. Davidson, Phys. Rev. ST Accel. Beams 6, 044401 (2003).

[16] D. D.-M. Ho, S. T. Brandon, and E. P. Lee, Part. Accel. 35, 15 (1991).

[17] H. Qin, C. Jun, R. C. Davidson, and P. Heitenroeder, in Proceedings of the 2001 Particle Accelerator Conference, Chicago (IEEE, Piscataway, NJ, 2001), p. 1761.

[18] E. P. Lee and J. J. Barnard, in Proceedings of the 2001 Particle Accelerator Conference, Chicago (Ref. [17]), p. 2929.

[19] M. J. L. de Hoon, E. P. Lee, J. J. Barnard, and A. Friedman, Phys. Plasmas 10, 855 (2003).

[20] M. Watanabe, M. Nakajima, M. Shiho, K. Horioka, K. Takayama, and J. Kishiro, Rev. Sci. Instrum. 73, 1756 (2002).

[21] H. Qin and R. C. Davidson, Phys. Rev. ST Accel. Beams 5, 034401 (2002).

[22] J. J. Barnard et al., Nucl. Instrum. Methods Phys. Res., Sect. A 415, 218 (1998), and references therein.

[23] A. I. Ogoyski, T. Someya, T. Sasaki, and S. Kawata, Phys. Lett. A 315, 372 (2003).

[24] T. Kikuchi, M. Nakajima, and K. Horioka, J. Plasma Fusion Res. 79, 105 (2003).
[25] T. Kikuchi, M. Nakajima, and K. Horioka, in Proceedings of the International Workshop on Recent Progress in Induction Accelerators, Tsukuba, Japan, 2002 (KEK Report No. 2002-30, 2003), p. 152.

[26] R. C. Davidson and H. Qin, Physics of Intense Charged Particle Beams in High Energy Accelerators (World Scientific, Singapore, 2001).

[27] T. Kikuchi, M. Nakajima, and K. Horioka, Laser Part. Beams 20, 589 (2002).

[28] S. M. Lund, O. Boine-Frankenheim, G. Franchetti, I. Hofmann, and P. Spiller, in Proceedings of the 1999 Particle Accelerator Conference, New York (IEEE, Piscataway, NJ, 1999), p. 1785.

[29] C. K. Birdsall and A. B. Langdon, Plasma Physics via Computer Simulation (McGraw-Hill, New York, 1985).

[30] R.W. Hockney and J.W. Eastwood, Computer Simulation Using Particles (McGraw-Hill, New York, 1981).

[31] M. Ikegami, Nucl. Instrum. Methods Phys. Res., Sect. A 454, 289 (2000).

[32] Q. Qian, R. C. Davidson, and C. Chen, Phys. Rev. E 51, R5216 (1995).

[33] S. Machida and M. Ikegami, in Proceedings of the Workshop on Space Charge Physics in High Intensity Hadron Rings, AIP Conf. Proc. No. 448 (AIP, New York, 1998), p. 73.

[34] K. Sakurai, T. Aoki, W. H. Lee, and K. Kato, Comput. Math. Applic. 43, 621 (2002).

[35] J. H. Ferziger and M. Perić, Computational Methods for Fluid Dynamics (Springer-Verlag, Berlin, 1996), p. 106.

[36] J. J. Barnard et al., Phys. Fluids B 5, 2698 (1993).

[37] M. G. Tiefenback and D. Keefe, IEEE Trans. Nucl. Sci. 32, 2483 (1985).

[38] Y. K. Batygin, in Proceedings of the Computational Accelerator Physics Conference, Los Alamos, 1993, AIP Conf. Proc. No. 297 (AIP, New York, 1994), p. 419.

[39] Y. K. Batygin, in Proceedings of the Space Charge Dominated Beams and Applications of High Brightness Beams, Bloomington, 1995, AIP Conf. Proc. No. 377 (AIP, New York, 1995), p. 290.

[40] M. Matsumoto and T. Nishimura, ACM Trans. Modeling Comput. Simulation 8, 3 (1998).

[41] I. Hofmann and J. Struckmeier, Part. Accel. 21, 69 (1987).

[42] J. Struckmeier and I. Hofmann, Part. Accel. 39, 219 (1992).

[43] W. H. Press, S. A. Teukolsky, W. T. Vetterling, and B. P. Flannery, Numerical Recipes in C: The Art of Scientific Computing (Cambridge University Press, New York, 1988), p. 377. 